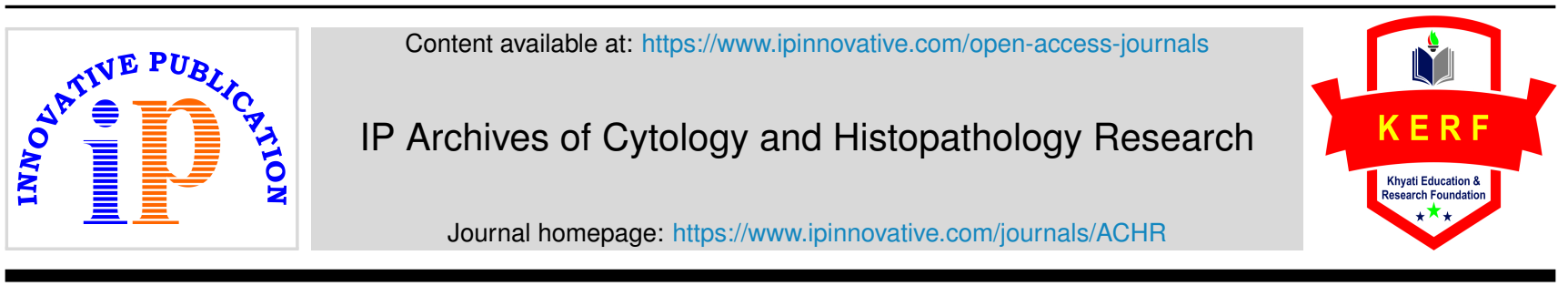

Original Research Article

\title{
Cytohistological correlation of salivary gland tumours with emphasis on Milan system for reporting: A novel step towards internal quality assurance
}

\author{
Anandraj Vaithy.K ${ }^{1}{ }^{*}$, ATM Venkat Raghavan ${ }^{1}$, E.S Keerthika Sri ${ }^{1}$, K.R Umadevi ${ }^{1}$ \\ ${ }^{1}$ Dept. of Pathology, Mahatma Gandhi Medical College\& Research Institute, Sri Balaji Vidyapeeth University (Deemed), \\ Puducherry, India
}

\section{A R T I C L E I N F O}

Article history:

Received 22-11-2020

Accepted 07-12-2020

Available online 30-12-2020

Keywords:

Milan system

Salivary gland carcinoma

Diagnostic histopathology

Cytology

\begin{abstract}
A B S T R A C T
Background: Fine needle aspiration cytology (FNAC) is a cost-effective diagnostic technique for evaluation of salivary gland lesions. But, then, cytological evaluation of salivary gland lesions also has lot of challenges. To overcome the difficulties, "The Milan system for reporting salivary gland cytopathology" (MSRSGC) was introduced for diagnosis and management and establishing the risk of malignancy (ROM) in different categories. The present study was conducted to grade the salivary gland lesions according to Milan system of reporting and to correlate with their histopathological findings.

Material and Methods : The current study was conducted in the department of Pathology, Mahatma Gandhi Medical College and Research Institute, Sri Balaji Vidyapeeth, Pondicherry for a period of 5 years. Around 153 salivary gland lesions were aspirated. Salivary gland swellings were examined clinically, correlated with the details on the request forms and ultrasound findings, palpated and aspirated. The cytological features were evaluated and categorized according to Milan System for Reporting Salivary Gland Cytopathology": Category 1: Nondiagnostic (ND); Category 2: Non-neoplastic (NN); Category 3: Atypia of undetermined significance (AUS); Category 4a: Neoplasm: benign (NB), Category 4b: Neoplasm: salivary gland neoplasm of uncertain malignant potential (SUMP); Category 5: suspicious of malignancy (SM); and Category 6: Malignant (M). They were further correlated with histopathological findings. All data were entered in MS excel sheet.

Results : Total 153 cases were evaluated cytologically, and histological followup was available in 134 cases. The distribution of cases into different categories were as follows Non-Diagnsotic (2.6\%), NonNeoplastic (20.9\%), Atypia of Undetermined Significance (1.9\%), Neoplastic-Benign (41.1\%),Salivary gland neoplasm of Uncertain Malignant Potential (0\%), Suspicious of Maligancy (0\%) and Malignancy $(33.3 \%)$. Risk of malignancy were observed in categories 2 and 6 and were $3.84 \%$ and $78.2 \%$. Sensitivity of FNAC in diagnosing salivary gland lesions was $84.2 \%$, specificity was $98.21 \%$,positive predictive value was $94.64 \%$, and negative predictive value was $91.70 \%$ with an accuracy of $93.60 \%$.

Conclusion: MSRSGC is a novel reporting system in cytological diagnosis of salivary gland lesions. Implementation of this reporting system in cytological diagnosis has enabled establishment of adequate diagnosis, assessment of risk stratification and facilitating clinicians to take further step in management plan.
\end{abstract}

(C) This is an open access article distributed under the terms of the Creative Commons Attribution License (https://creativecommons.org/licenses/by/4.0/) which permits unrestricted use, distribution, and reproduction in any medium, provided the original author and source are credited.

\section{Introduction}

Fine needle aspiration cytology (FNAC) of salivary gland plays an important role in the diagnosis of salivary gland tumors. It is a minimally invasive, safe diagnostic modality,

\footnotetext{
* Corresponding author.

E-mail address: kanandrv@gmail.com (A. Vaithy.K).
}

which fetches the surgeons to take a further step of management. There are several studies that have proven that FNAC is sensitive test in distinguishing neoplastic from non-neoplastic salivary gland lesions. The sensitivity of FNAC ranges from $86 \%$ to $100 \%$, and specificity ranges between $90 \%-100 \%$. FNAC also helps to arrive at a 
diagnosis of primary versus metastatic tumor. ${ }^{1}$

The 2017 World Health Organization (WHO) classification introduces 30 different epithelial salivary gland neoplasms. Majority of the tumors are benign with predominant location being parotid and submandibular glands. Amongst the benign salivary gland tumors, $65 \%$ are Pleomorphic adenoma and amongst malignant salivary gland tumors, Mucoepidermoid carcinoma being the most common. New entities like Secretory Carcinoma, Sclerosing polycystic adenosis are been added. Few entities are modified with names like Polymorphous.

Adenocarcinoma, (Formerly polymorphous low-grade Adenocarcinoma), Intraductal carcinoma (Formerly low grade cribriform cystadenocarcinoma, low grade salivary duct carcinoma, salivary duct carcinoma in situ) and poorly differentiated carcinoma. Although, FNAC has advantages, there are few challenges that a cytologist encounters in diagnosing a salivary gland lesion.

With introduction of certain systems of reporting, a reasonable cytological diagnosis is made, which provides risk stratification and enabling the surgeon to take a further step in management plan. ${ }^{2}$ The American Society of Cytopathology and International Academy of Cytology came up with a tiered international classification scheme called the "Milan System for Reporting Salivary Gland Cytopathology" (MSRSGC). The MSRSGC is a six tier classification comprising the following categories: I-Nondiagnostic, II-Non-neoplastic, III-Atypia of Undetermined significance (AUS), IV-Neoplasm-benign, Salivary Gland Neoplasm of Uncertain Malignant Potential (SUMP), VSuspicious for Malignancy, VI-Malignant. ${ }^{3}$

The present study was conducted to grade the salivary gland lesions according to Milan system of reporting and to correlate with their histopathological findings.

\section{Materials and Methods}

The current study was conducted in the department of Pathology, Mahatma Gandhi Medical College and Research Institute, Sri Balaji Vidyapeeth, Pondicherry for a period of 5 years. Around 153 salivary gland lesions were aspirated. Salivary gland swellings were examined clinically, correlated with the details on the request forms and ultrasound findings, palpated and aspirated by 22 or 23 gauze needle via manual or ultrasound guided using negative suction technique. The material obtained were smeared on glass slides by trained cytopathologists and stained with Hematoxylin and Eosin (H\&E), Pap stain and May-Grunwald Giemsa (MGG) stains. For staining with H \& E and Pap stains, slides were fixed in $95 \%$ ethanol for a period of 30 minutes before the staining whereas, for MGG stain, smears were air-dried. The cytological features were evaluated and categorized according to Milan System for Reporting Salivary Gland Cytopathology" (MSRSGC). The cytological features were correlated with the histopathological features on biopsy specimens. Specimens sent in $10 \%$ Neutral Buffered Formalin, were fixed overnight, grossed the next day correlating with all the patient details and clinical and radiological findings on the request forms, gross features were noted and multiple bits were taken from the representative areas and were subjected to tissue processing using automated tissue processor. Sections were then taken and stained with H\&E. Final histopathological report was given after the hierarchical pattern of reporting. All the data obtained including patient name, registration number, age, sex, clinical diagnosis, cytological and histopathological diagnosis were entered in MS excel sheet.

\section{Results}

A total of 153 patients underwent Fine Needle Aspiration Cytology (FNAC), out of which predominant patients were males than females and M:F ratio being 1.1:1. The age distribution of salivary gland lesions is given below in the form of Graph 1. Majority of cases were detected in the age group between $21-40$ years, accounting to 84 cases $(56 \%)$, least affected age group is below 20 years accounting to 4 cases(3\%).

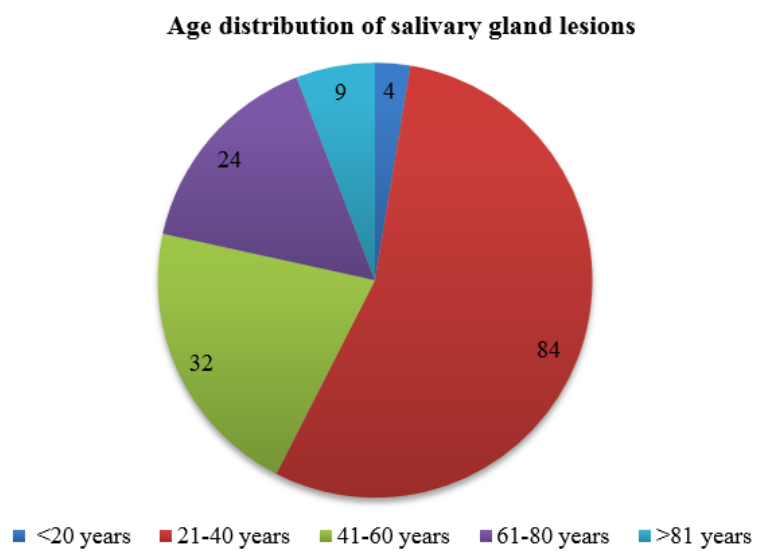

Graph 1: Age distribution of salivary gland lesions

Parotid gland was involved in 63\% cases, followed by submandibular gland in $27.3 \%$ with minor salivary gland involved in $9.7 \%$ of cases.

The FNAC distribution of cases according to MSRSGC is shown in Table 1. Category 4 a (neoplasm-benign) was reported in largest number, followed by category 6 (Malignant), accounting to $33.3 \%$ and the least reported was category $4 \mathrm{~b}$ (Salivary Gland Neoplasm of Uncertain Malignant Potential (SUMP) and category 5(Suspicious for Malignancy).

Histological follow up was available in 134 cases, and available histopathological comparison according to MSRSGC and is shown in the above table. In category 1 (Non-Diagnostic) out of 4, follow up was available in only 
Table 1: FNAC distribution of MSRSGC

\begin{tabular}{|c|c|c|c|c|c|c|c|c|}
\hline Category & Cat 1 & Cat 2 & Cat 3 & Cat $4 a$ & Cat 4b & Cat 5 & Cat 6 & Total \\
\hline No. of cases & $4(2.6 \%)$ & $32(20.9 \%)$ & $3(1.9 \%)$ & $63(41.1 \%)$ & $0(0 \%)$ & $0(0 \%)$ & $51(33 \%)$ & 153 \\
\hline $\begin{array}{l}\text { No. of cases with histological } \\
\text { follow up }\end{array}$ & 2 & 26 & 1 & 59 & 0 & 0 & 46 & 134 \\
\hline Non-neoplastic & 1 & 24 & 1 & 11 & 0 & 0 & 6 & 43 \\
\hline Neoplastic & 1 & 1 & 0 & 48 & 0 & 0 & 4 & 55 \\
\hline Malignant & 0 & 1 & 0 & 0 & 0 & 0 & 36 & 36 \\
\hline Risk of malignancy & $0(0 \%)$ & $\begin{array}{c}1 / 26 \\
(3.84 \%)\end{array}$ & $0(0 \%)$ & $0(0 \%)$ & $0(0 \%)$ & $0(0 \%)$ & $\begin{array}{c}36 / 46 \\
(78.2 \%)\end{array}$ & $\begin{array}{c}36 / 134 \\
(26.8 \%)\end{array}$ \\
\hline
\end{tabular}

2 cases, and out of these, 1 case turned out to be chronic sialadenitis and another one as myoepithelioma.

In category 2 (NonNeoplastic), histopathological followup of 26 cases were available; out of total 26 cases, 1 case of benign tumor was reported. One was pleomorphic adenoma, which was wrongly diagnosed as category 2 (Non Neoplastic)- chronic sialadenitis. Another one case was reported as epithelial inclusion cyst on cytology and tuned out to be high grade mucoepidermoid carcinoma. Risk of malignancy accounted to $3.84 \%$.Out of 24 non-neoplastic lesions, 17 were reported chronic sialadenitis, 5 reported as acute suppurative sialadenitis and two were reported as lymphoepithelial cyst.

Histological follow up of 1 out of 3 cases were available in category 3 (Atypia of Undetermined significance) and was reported as cellular pleomorphic adenoma.

Category $4 \mathrm{a}$ had histological follow up of 59 cases out of 63 cases. 39 cases were diagnosed as pleomorphic adenoma and showed similar concordance on histopathology, 1 case which was diagnosed as pleomorphic adenoma on cytology, turned out be a metastatic papillary carcinoma, 4 cases were diagnosed as warthins tumor, 2 cases of myoepithelioma and 2 cases of basal cell adenoma were reported.11 cases, which were reported as benign salivary gland tumor, turned out to be chronic sialadenitis on histopathology.

There were no cases reported in Category $4 \mathrm{~b}$ and 5. Category 6 is for the smears that are diagnostic for malignant lesion, and histological follow up of 46 cases was available.

Six cases turned up to be non-neoplastic lesions, out of which 4 cases were chronic sialadenitis and two were lymphoepithelial cysts. 4 cases turned out be benign tumors, out of which three were diagnosed as cellular pleomorphic adenoma and one as basal cell adenoma in histopathology. Other 36 cases in histopathology showed concordance with cytology, out of which 17 cases were reported as Mucoepidermoid carcinoma, 8 cases were reported as acinic cell carcinoma, 6 cases were reported as adenoid cystic carcinoma, 3 cases of pleomorphic ex sarcoma and one case of metastatic papillary carcinoma were reported. Risk of malignancy in category 6 accounted to $78.2 \%$.

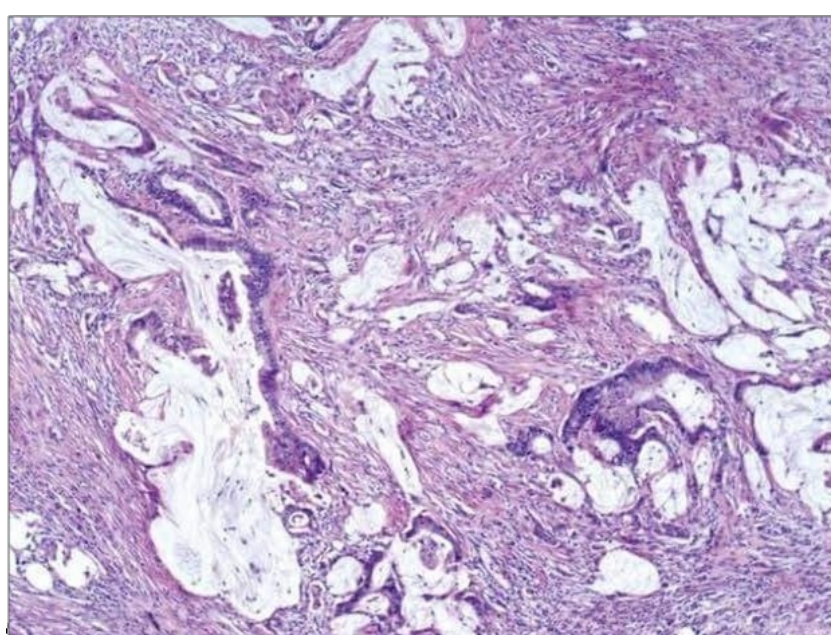

Fig. 1: Histomorphology of Muco-epidermoid carcinoma of salivary gland-High grade, $\mathrm{H} \& \mathrm{E}, 40 \mathrm{X}$

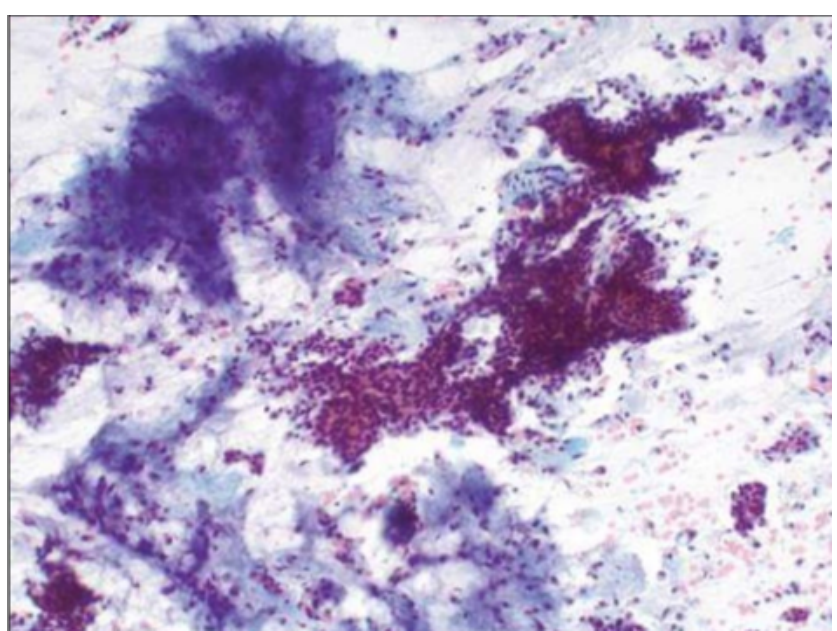

Fig. 2: Cytomorphology of pleomorphic adenoma showing classical chondromyxoid background, Pap 10X 


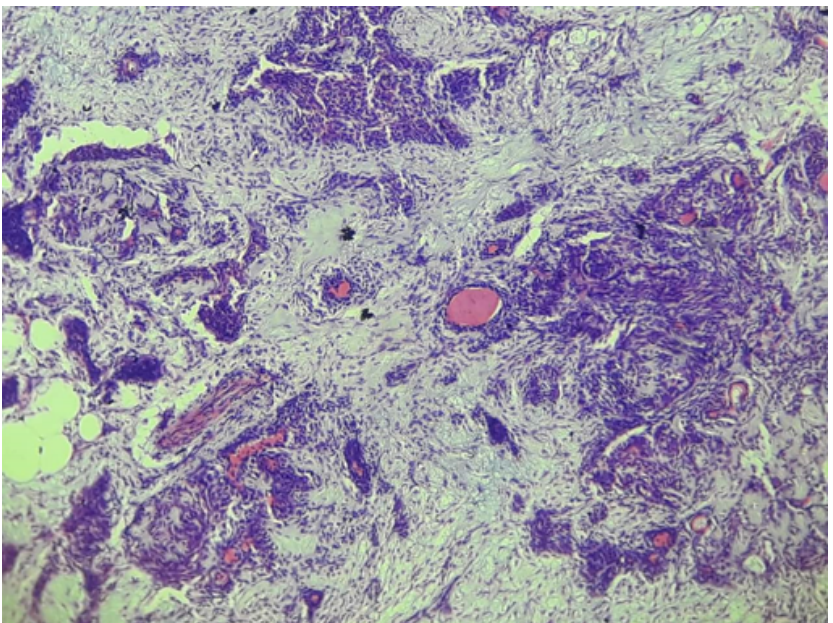

Fig. 3: Histomorphology of Pleomorphic adenoma of salivary gland, $H \& E, 40 x$

\section{Discussion}

FNAC is a safe, accurate, and cost-effective diagnostic technique in diagnosis and management of salivary gland lesions.MSRSGC is a novel reporting system in the diagnosis of salivary gland lesions, thereby enabling proper and appropriate diagnosis and facilitating the clinicians to take further step in management plan. ${ }^{1,4}$

It classified FNAC into six categories; ND (Nondiagnostic), NN (Non-neoplastic), AUS (Atypia of undetermined significance), NB (Neoplastic-benign), SUMP (salivary gland neoplasm, of uncertain neoplasm), $\mathrm{SM}$ (Suspicious of malignancy), and malignant with Risk of malignancy in

Category 2 and category 6 being $3.84 \%$ and $78.2 \%$. There are other studies that have provided risk of stratification in salivary gland lesions, study of kala et al showed the following findings: category $1-25 \%$,category 2- $5 \%$, category $3-20 \%$, category $4 a-4.4 \%$, category $4 b-$ $33.3 \%$, category $5-85.7 \%$, and category $6-97.5 \% .{ }^{5}$ The present study had also categorized salivary gland FNAC into six categories according to MSRSGC, highest risk of stratification is in the category 6 in both the studies, although percentages are different. In the present study, risk of stratification is $0 \%$ in other categories like $1,3,4 \mathrm{a}, 4 \mathrm{~b}$ and 5 because, the slides are been viewed by consultants in an hierarchical pattern, thereby avoiding diagnostic pitfalls.

Category 1 cases are non-diagnostic salivary gland lesions. There were 4 cases $(2.6 \%)$ in Nondiagnostic category and on histological follow up, 2 cases that were diagnosed as Nondiagnostic in FNAC were reclassified as chronic sialadenitis, and the presence of marked fibrosis and loss of acini might be the possible reason for the false diagnosis. Another case was diagnosed as myoepithelioma on histopathology. In this case, due to the cystic change of the tumor, acellular aspirate from cystic areas during FNAC has lead to diagnosis as category 1.

Under category 2 (Non Neoplastic), histopathological follow-up of 26 cases were available; out of total 26 cases, 1 case of benign tumor was reported. One was pleomorphic adenoma, which was wrongly diagnosed as category 2 (Non Neoplastic) - chronic sialadenitis. This was because the tumor has elicited a chronic inflammatory response, which showed lymphoplasmacytic and histiocytic infiltration on FNAC. Another one case was reported as epithelial inclusion cyst on cytology and tuned out to be high grade mucoepidermoid carcinoma (Figure 1). The cystic change of the tumor and the presence of more number of squamous cells led to the misdiagnosis of epithelial inclusion cyst on FNAC. Out of 24 nonneoplastic lesions, 17 were reported chronic sialadenitis, 5 reported as acute suppurative sialadenitis and two were reported as lymphoepithelial cyst. Studies of Kala et al and Katta et al encountered similar kind of presentation of mucoepidermoid carcinoma on FNAC. Our study correlates with the studies of Kala et al and Katta et al. ${ }^{5,6}$

Histological follow up of 1 out of 3 cases were available in category 3 (Atypia of Undertermined significance) and was reported as cellular pleomorphic adenoma. This was misdiagnosed as category 3 on FNAC because the presence of increased cellularity of ductal epithelial cells mimicked atypia. Study of Katta et al is in concordance of our study, as the former study also reported one case under category 3. Studies of Kala et al ,Katta et al and Karuna et al also more or less correlate with our study, except in the number of cases. ${ }^{5-7}$

Category 4a had histological follow up of 59 cases out of 63 cases. 39 cases were diagnosed as pleomorphic adenoma and showed similar concordance on histopathology. (Figures 2 and 3). 4 cases were diagnosed as Warthins tumor, 2 cases of myoepithelioma and 2 cases of basal cell adenoma were reported. 1 case which was diagnosed as pleomorphic adenoma on cytology, turned out be a metastatic papillary carcinoma. This was because the papillary pattern of cell arrangement mimicked the fibromyxoid background of Pleomorphic adenoma.11 cases, which were reported as benign salivary gland tumor, turned out to be chronic sialadenitis on histopathology. The highly cellular aspirate and presence of fibroblasts has led to misdiagnosis of benign salivary gland tumor on FNAC. Our present study more or less correlated with the studies of Kala et al, Katta et al and Karuna et al. ${ }^{5-7}$

There were no cases reported in Category $4 \mathrm{~b}$ and 5. Category 6 is for the smears that are diagnostic for malignant lesion, and histological follow up of 46 cases was available.

Six cases turned up to be non-neoplastic lesions, out of which 4 cases were chronic sialadenitis and two were lymphoepithelial cysts. This was because of cellular atypia on FNAC, which led to categorize under malignancy. ${ }^{3}$ 
cases turned out be benign tumors, out of which three were diagnosed as cellular pleomorphic adenoma and one as basal cell adenoma in histopathology. This is because cellular Pleomorphic adenoma and basal cell adenoma can be misinterpreted as malignant lesions on cytology due to the higher cellularity. Other 36 cases in histopathology showed concordance with cytology. Our present study more or less correlated with the studies of Kala et al, Katta et al and Karuna et al. ${ }^{5-7}$

FNAC is an effective tool to distinguish benign and malignant neoplasm with high specificity $98 \% .{ }^{8}$ However, sometimes it is very difficult to differentiate between a benign neoplasm and low grade malignant neoplasm. However, our study predominantly showed concordant results on both cytology and histopathology. 9 Sensitivity of FNAC in diagnosing salivary gland lesions was 84.2 $\%$, specificity was $98.21 \%$, positive predictive value was $94.64 \%$, and negative predictive Value was $91.70 \%$ with an accuracy of $93.60 \%$.

\section{Conclusion}

MSRSGC is a novel reporting system which had led to systematic approach in diagnosis of salivary gland lesions and thereby facilitating effective role for the clinicians to take further step in management plan. Thus, FNAC is a costeffective and sensitive diagnostic technique in diagnosis of salivary gland lesions. But, without histopathological evaluation, diagnosis of a salivary gland lesion remains incomplete. ${ }^{10}$

\section{Source of Funding}

No financial support was received for the work within this manuscript.

\section{Conflict of Interest}

The authors declare they have no conflict of interest.

\section{References}

1. Colella G, Cannavale R, Flamminio F, Foschini MP. Fine-Needle Aspiration Cytology of Salivary Gland Lesions: A Systematic Review. Journal of Oral and Maxillofacial Surgery. 2010;68(9):21462153. Available from: https://dx.doi.org/10.1016/j.joms.2009.09.064. do1:10.1016/].10ms.2009.09.064

2. Chakrabarti S, Bera M, Bhattacharya PK, Chakrabarty D, Manna AK Pathak S. Study of salivary gland lesions with fine needle aspiration cytology and histopathology along with immunohistochemistry. $J$ IndianMed Assoc. 2010;108:833-839.
3. Rohilla M, Singh P, Rajwanshi A, Gupta N, Srinivasan R, Dey $\mathrm{P}$, et al. Three-year cytohistological correlation of salivary gland FNA cytology at a tertiary center with the application of the Milan system for risk stratification. Cancer Cytopathology. 2017;125(10):767-775. Available from: https://dx.doi.org/10.1002/ cncy. 21900. do1:10.1002/cncy.21900.

4. Rossi ED, Faquin WC, Baloch Z, Barkan GA, Foschini MP, Pusztaszeri M, et al. The Milan System for Reporting Salivary Gland Cytopathology: Analysis and suggestions of initial survey. Cancer Cytopathology. 2017;125(10):757-766. Available from: https://dx. doi.org/10.1002/cncy.21898. do1:10.1002/cncy.21898.

5. Kala C, Kala S, Khan L. Milan system for reporting salivary gland cytopathology: An experience with the implication for risk of malignancy. Journal of Cytology. 2019;36(3):160 160. Available from: https://dx.doi.org/10.4103/joc.joc_165_18. do1:10.4103/10c.joc 16518

6. Katta R, Chaganti DP. Application of the Milan system of reporting salivary cytopathology-A retrospective cytohistological correlation study. J NTR Univ Health Sci. 2019;8:11-18.

7. Karuna V, Gupta P, Rathi M, Grover K, Nigam J, Verma N. Effectuation to Cognize malignancy risk and accuracy of fine needle aspiration cytology in salivary gland using "Milan System for Reporting Salivary Gland Cytopathology": A 2 years retrospective study in academic institution. Indian Journal of Pathology and Microbiology. 2019;62(1):11-11. Available from: https://dx.doi.org/ 10.4103/ijpm.ijpm_380_18. do1:10.4103/1]pm.1]pm_380_18.

8. Song IH, Song JS, Sung CO, Rohm JL, Choi SH, Nam SY. Accuracy of core needle biopsy versus fine needle aspiration cytology for diagnosing salivary gland tumors. J PatholTransl Med. 2015;49:136179 .

9. Tyagi R, Dey P. Diagnostic problems of salivary gland tumors. Diagnostic Cytopathology. 2015;43(6):495-509. Available from: https://dx.doi.org/10.1002/dc.23255. doi:10.1002/dc.23255.

10. Mairembam P, Jay A, Beale T, Morley S, Vaz F, Kalavrezos $\mathrm{N}$, et al. Salivary gland FNA cytology: role as a triage tool and an approach to pitfalls in cytomorphology. Cytopathology. 2016;27(2):91-96. Available from: https://dx.doi.org/10.1111/cyt. 12232. do1:10.1111/cyt.12232

\section{Author biography}

Anandraj Vaithy.K, Associate Professor

ATM Venkat Raghavan, Assistant Professor

E.S Keerthika Sri, Tutor

K.R Umadevi, Professor

Cite this article: Vaithy.K A, Venkat Raghavan ATM, Sri ESK

Umadevi KR. Cytohistological correlation of salivary gland tumours

with emphasis on Milan system for reporting: A novel step towards

internal quality assurance. IP Arch Cytol Histopathology Res

2020;5(4):283-287. 